\title{
Identification of modifiable risk factors for acute kidney injury after coronary artery bypass graft surgery in an Asian population
}

\author{
Roderica Rui Ge Ng, ${ }^{a}$ Sophia Tsong Huey Chew, MBBS, MMed, ${ }^{\text {b,c }}$ Weiling Liu, BSc(Hons), \\ Liang Shen, PhD, ${ }^{a}$ and Lian Kah Ti, MBBS, MMed ${ }^{\mathrm{a}, \mathrm{d}}$
}

\begin{abstract}
Objective: Postoperative acute kidney injury (AKI) after cardiopulmonary bypass (CPB) with coronary artery bypass grafting is common and increases patient morbidity and mortality. Studies have identified the lowest hematocrit during $\mathrm{CPB}$, preoperative anemia, and intraoperative transfusion as modifiable AKI risk factors. Because Asians are smaller in body size, the use of standard CPB circuits can result in excessive hemodilution and subsequent transfusion to maintain the desired hematocrit target of $\geq 21 \%$ during CPB. Thus, we aimed to ascertain whether the lowest hematocrit during CPB, preoperative anemia, and intraoperative transfusion remained as independent modifiable risk factors associated with AKI in our prospective cohort of Asians.
\end{abstract}

\begin{abstract}
Methods: Data from 1448 patients who had undergone coronary artery bypass grafting with CPB from December 2008 to December 2010 at Singapore's 2 national heart centers were obtained. The perioperative risk factors were analyzed for their associations with postoperative AKI. AKI was defined using the Acute Kidney Injury Network stage 1 criteria.

Results: The incidence of AKI was $27.0 \%$ and mean lowest hematocrit during CPB was $24.5 \% \pm 3.8 \%$. The risk of AKI increased with a decreasing lowest hematocrit during CPB (relative risk, $0.933 ; 95 \%$ confidence interval, $0.899-0.968 ; P<.001$ ), in particular with the lowest hematocrit of $\leq 22 \%$. A $23 \%$ increased risk of AKI was found for preoperative anemia (relative risk, 1.225 ; $95 \%$ confidence interval, 1.022-1.468; $P=.028)$. Intraoperative transfusion was related on univariate analysis $(P<.001)$ but was not independently associated on multivariate analysis (relative risk, $0.961 ; 95 \%$ confidence interval, $0.782-1.180 ; P=.702$ ).
\end{abstract}

Conclusions: The lowest hematocrit and preoperative anemia were potentially modifiable risk factors independently associated with AKI after cardiac surgery in our Asian population. Blood transfusion did not affect the development of AKI in our population. (J Thorac Cardiovasc Surg 2014;147:1356-61)

Postoperative acute kidney injury (AKI) after coronary artery bypass grafting (CABG) with cardiopulmonary bypass $(\mathrm{CPB})$ is common and increases patient morbidity and mortality. ${ }^{1,2}$ The perioperative risk factors for AKI include patient age, history of diabetes, intra-aortic balloon pump use, and CPB duration. Factors, such as the lowest hematocrit during $\mathrm{CPB}$, intraoperative red blood cell (RBC) transfusion, and preoperative anemia, have been identified

From the National University of Singapore Yong Loo Lin School of Medicine, Singapore; Department of Anesthesia and Surgical Intensive Care, ${ }^{\mathrm{b}}$ Singapore General Hospital, Singapore; Department of Cardiovascular and Metabolic Disorders, ${ }^{c}$ Duke-National University of Singapore Graduate Medical School, Singapore; and Department of Anesthesia, ${ }^{\mathrm{d}}$ National University Health System, Singapore.

This research was supported by a Khoo Research grant (grant R-913-200-023-304) from the Duke-National University of Singapore Graduate Medical School, Singapore.

Disclosures: Authors have nothing to disclose with regard to commercial support. Received for publication June 19, 2013; revisions received Aug 14, 2013; accepted for publication Sept 13, 2013; available ahead of print Nov 4, 2013.

Address for reprints: Lian Kah Ti, MBBS, MMed, Department of Anesthesia, National University Hospital, 5 Lower Kent Ridge Rd, Singapore 119074 (E-mail: lian_kah_ti@nuhs.edu.sg).

0022-5223/\$36.00

Copyright (c) 2014 by The American Association for Thoracic Surgery

http://dx.doi.org/10.1016/j.jtcvs.2013.09.040 as independent modifiable risk factors that could contribute to postoperative AKI. . $^{3-8}$

The lowest acceptable hematocrit during CPB has not been definitively identified. However, it is generally agreed that a hematocrit of $\geq 21 \%$ during CPB decreases renal complications. $^{4,9}$ The current practice has been to maintain the hematocrit at or $>21 \%$ during $\mathrm{CPB}$. This can require blood transfusion. However, the transfusion of blood to ameliorate the effects of hemodilution can increase the risk of AKI. ${ }^{3,4,6}$

Preoperative anemia has been identified to be the single most important determinant of intraoperative blood transfusion. ${ }^{10}$ Karkouti and colleagues ${ }^{11}$ have shown that anemic patients are most likely to require multiple blood transfusions during cardiac surgery and are also most likely to be harmed by them. Preoperative anemia has therefore been shown to increase the risk of AKI. ${ }^{6,7}$

Our patient population was predominantly Asian, who are typically shorter and lighter than our Western counterparts. ${ }^{12}$ Using Nadler's formula, the total blood volume is related to the cubed product of the height and directly to the weight. ${ }^{13}$ Therefore, far greater hemodilution can occur in our patients from the fixed amount of blood-free prime in the CPB circuit, resulting in a lower hematocrit during $\mathrm{CPB}$. This can 


$$
\begin{aligned}
& \text { Abbreviations and Acronyms } \\
& \begin{aligned}
\mathrm{AKI} & =\text { acute kidney injury } \\
\mathrm{CABG} & =\text { coronary artery bypass grafting } \\
\mathrm{CI} & =\text { confidence interval } \\
\mathrm{CPB} & =\text { cardiopulmonary bypass } \\
\mathrm{RBC} & =\text { red blood cell } \\
\mathrm{RR} & =\text { relative risk }
\end{aligned}
\end{aligned}
$$

necessitate a greater rate of intraoperative transfusion to conform to the hematocrit target, often requiring transfusions even for patients who were not anemic preoperatively.

We postulated that the smaller body sizes of our patients would compound the effect of preoperative anemia and increase the rate of transfusion, leading to postoperative AKI. We therefore embarked on the present study to determine the incidence of AKI after CPB in our patient population and to study the effect of known modifiable risk factors (ie, lowest hematocrit during $\mathrm{CPB}$, preoperative anemia, and $\mathrm{RBC}$ transfusion) on the incidence of AKI after CABG.

\section{METHODS}

\section{Study Design, Database, and Population Selection}

With institutional review board approval, we prospectively followed up 1744 Asian patients who had undergone primary, isolated CABG from December 2008 to October 2011 at Singapore's 2 national heart centers (National University Health System and Singapore General Hospital). All patients provided written informed consent. The perioperative variables were collated and maintained in a cardiac database with regular audits. A total of 296 patients were excluded from the present study because they had had preoperative renal impairment $(\mathrm{n}=237)$, had missing preoperative and postoperative creatinine values $(\mathrm{n}=25)$, or undergone off-pump surgery $(\mathrm{n}=34)$. The total number of patients who met the inclusion and exclusion criteria was 1448 . The normal creatinine range provided by our laboratories was 60 to $105 \mu \mathrm{mol} / \mathrm{L}$ for men and 40 to $75 \mu \mathrm{mol} / \mathrm{L}$ for women. Renal impairment was defined as serum creatinine $>10 \mu \mathrm{mol} / \mathrm{L}$ greater than normal and/or dialysis. This corresponded to the cutoff values of serum creatinine of $115 \mu \mathrm{mol} / \mathrm{L}$ and $90 \mu \mathrm{mol} / \mathrm{L}$ that have been validated in men and women, respectively, in screening for early renal impairment. ${ }^{14}$

\section{Renal Function Assessment and Perioperative Renal Data}

The preoperative serum creatinine value used was that obtained closest to surgery, typically at preadmission testing within 1 week of surgery. If a material change had occurred in the patient's condition, the serum creatinine measurement would be repeated the day before surgery, and that value would be taken as the preoperative serum creatinine. The peak serum creatinine was the highest creatinine value obtained within the first 3 postoperative days. Stage I of the Acute Kidney Injury Network criteria was used as the primary outcome, defined as an absolute increase in serum creatinine of $\geq 26.4$ $\mu \mathrm{mol} / \mathrm{L}$ and $/$ or a $50 \%$ increase in the serum creatinine to the peak serum creatinine from the preoperative serum creatinine. The creatinine levels typically peaked on postoperative day 2 and had returned to baseline by day $5 .{ }^{15}$

\section{Hematocrit, Hemodynamic, and Transfusion Data}

The hematocrit was measured at heparinization and every 20 minutes during CPB. Testing was performed using the routine arterial blood gas analysis protocols on the Siemens RAPIDlab 1265 (Siemens Healthcare Diagnostics, Tarrytown, NY). Blood transfusion was given to maintain a hematocrit of $\geq 21 \%$ during CPB and was guided by institutional protocol.

\section{Preoperative Anemia Data}

Preoperative anemia was defined according to the World Health Organization's gender-based definition of $<12.0 \mathrm{~g} / \mathrm{dL}$ for women and $<13.0$ $\mathrm{g} / \mathrm{dL}$ in men.

\section{Perioperative Anesthesia and Surgical and Perfusion Management}

The perioperative surgical management and clinical practices at both institutions were similar and followed international practice. Typically, anesthesia was induced with intravenous induction agents (etomidate or propofol) and maintained with a balanced anesthesia regimen of lowdose fentanyl (10-20 $\mu \mathrm{g} / \mathrm{kg}$ ) and volatile agents (primarily sevoflurane). Conventional CPB circuits with roller pumps, membrane oxygenators, heat exchangers, venous reservoirs, cardiotomy suction, and arterial blood filters were used. The volume of prime used in the CPB circuits was typically 1300 to $1400 \mathrm{~mL}$. The perfusion targets were mild-to-moderate hypothermia $\left(32^{\circ}-35^{\circ} \mathrm{C}\right)$, hematocrit levels of $\geq 21 \%$, an activated clotting time of $>400$ seconds, a glucose level of $<10 \mathrm{mmol} / \mathrm{L}$, a nonpulsatile flow rate of 2.2 to $2.4 \mathrm{~L} / \mathrm{min} / \mathrm{m}^{2}$, and mean arterial pressure of 50 to $70 \mathrm{~mm} \mathrm{Hg}$. Myocardial protection was achieved with cold blood cardioplegia. Aprotinin was not used in any of the patients.

\section{Statistical Analysis}

Population demographics, medical history, preoperative risk assessment, intraoperative variables, and postoperative outcomes were analyzed descriptively. Univariate analysis was performed using a 2-tailed unpaired $t$ test or Mann-Whitney $U$ test for numerical factors after normality assumption was checked. The chi-square test was performed for categorical factors on univariate analysis. The factors from the univariate analysis with $P<.1$ were included in the multivariate model. Multivariate analysis was performed using the Poisson regression model with robust estimator to estimate the adjusted relative risk (RR) of developing AKI after CPB for $\mathrm{CABG}$ patients. The interaction effect between the lowest hematocrit during $\mathrm{CPB}$ and the use of transfusion and the presence of preoperative anemia was checked. Only the main effects would be included in the model if no significant interaction was present. Collinearity among the factors was also checked. All statistical analyses were performed using IBM SPSS, version 20.0 (IBM, Armonk, NY). The data were plotted and logarithmic trend lines drawn using Microsoft Excel 2010 (Microsoft, Redmond, Wash).

\section{RESULTS}

A total of 1744 Asian patients underwent CABG during the study period. Of these, 1448 patients met the inclusion and exclusion criteria for our study. Of the 1448 patients, 391 developed AKI postoperatively. These patients were more likely to be older and to have a history of diabetes and hypertension. Other risk factors included preoperative anemia, high preoperative creatinine, poor heart function, the use of loop diuretics, urgent or emergent CABG surgery, intra-aortic balloon pump use, a lower European System for Cardiac Operative Risk Evaluation (EuroSCORE) logistic score, longer $\mathrm{CPB}$ time, longer aortic crossclamp time, and greater number of vein grafts harvested. They were also more likely to have a lower lowest hematocrit during $\mathrm{CPB}$ and to have received RBC transfusion (Table 1). 
TABLE 1. Univariate analysis of perioperative risk factors associated with AKI

\begin{tabular}{lccr}
\hline \multicolumn{1}{c}{ Variable } & $\begin{array}{c}\text { AKI } \\
(\mathbf{n}=\mathbf{3 9 1})\end{array}$ & $\begin{array}{c}\text { No AKI } \\
(\mathbf{n}=\mathbf{1 0 5 7})\end{array}$ & $\boldsymbol{P}$ value \\
\hline Preoperative & & & \\
Age (y) & $61.7 \pm 9.4$ & $58.7 \pm 8.9$ & $<.001$ \\
Male gender & $326(83.4)$ & $897(84.9)$ & .488 \\
Body mass index $\left(\mathrm{kg} / \mathrm{m}^{2}\right)$ & $25.6 \pm 4.2$ & $25.1 \pm 3.7$ & .039 \\
History of diabetes mellitus & $219(56.0)$ & $517(48.9)$ & .016 \\
History of hypertension & $337(86.2)$ & $810(76.6)$ & $<.001$ \\
Preoperative anemia & $145(37.1)$ & $239(22.6)$ & $<.001$ \\
Preoperative creatinine & $91(30)$ & $83(25)$ & $<.001$ \\
$\quad(\mu$ mol/L) & & & \\
Loop diuretic medication & $67(17.1)$ & $114(10.8)$ & .001 \\
LVEF $<30 \%$ & $48(12.3)$ & $76(7.2)$ & .002 \\
Urgent or emergent surgery & $62(15.9)$ & $111(10.5)$ & .005 \\
Use of IABP & $79(20.2)$ & $96(9.1)$ & $<.001$ \\
Logistic EuroSCORE & $4.0 \pm 4.7$ & $2.9 \pm 3.3$ & $<.001$ \\
Intraoperative & & & \\
Lowest hematocrit during & $23.3 \pm 3.6$ & $24.9 \pm 3.8$ & $<.001$ \\
$\quad$ CPB (\%) & & & \\
RBC transfusion & $167(42.7)$ & $275(26.0)$ & $<.001$ \\
CPB time (min) & $128 \pm 77.6$ & $105.3 \pm 42.1$ & $<.001$ \\
Aortic crossclamp time (min) & $69.3 \pm 40.4$ & $62.6 \pm 32.6$ & .002 \\
Vein grafts harvested (n) & $3.1 \pm 0.8$ & $3 \pm 1$ & .038 \\
Use of left internal mammary & $380(97.2)$ & $1014(96.1)$ & .330 \\
$\quad$ artery & & & \\
\hline
\end{tabular}

Data presented as $\mathrm{n}(\%)$ or mean \pm standard deviation. AKI, Acute kidney injury; $L V E F$, left ventricular ejection fraction; $I A B P$, intra-aortic balloon pump; $R B C$, red blood cell; $C P B$, cardiopulmonary bypass; EuroSCORE, European System for Cardiac Operative Risk Evaluation.

\section{Lowest Hematocrit During CPB}

The mean lowest hematocrit for the whole cohort was $24.5 \% \pm 3.8 \%$ (range, $14 \%-47 \%$ ). The lowest hematocrit was $<21 \%$ in 192 patients ( $13.3 \%$ of the study population). These patients had an AKI incidence of $46.4 \%$ versus $24.0 \%$ for those whose hematocrit during CPB was $\geq 21 \%(P<.001)$. After adjusting for other statistically significant factors, the lowest hematocrit during $\mathrm{CPB}$ was independently associated with AKI (RR, $0.933 ; 95 \%$ confidence interval $[\mathrm{CI}], 0.899-0.968 ; P<.001)$. The incidence of AKI increased with a decreasing lowest hematocrit during $\mathrm{CPB}$, in particular, with the lowest hematocrit of $\leq 22 \%$ (Figure 1).

\section{Preoperative Anemia}

A total of 384 patients ( $26.5 \%$ of study population) had preoperative anemia, and $37.8 \%$ of these patients developed AKI. Preoperative anemia was also found to be independently associated with AKI (RR, 1.225; 95\% CI, $1.022-1.468, P=.028$ ). The presence of preoperative anemia incurred a $23 \%$ increased risk of AKI. The mean lowest hematocrit during CPB differed significantly between those with preoperative anemia who did and did not develop AKI $(21.6 \% \pm 2.6 \%$ vs $22.6 \% \pm 2.8 \% ; P=.001)$.

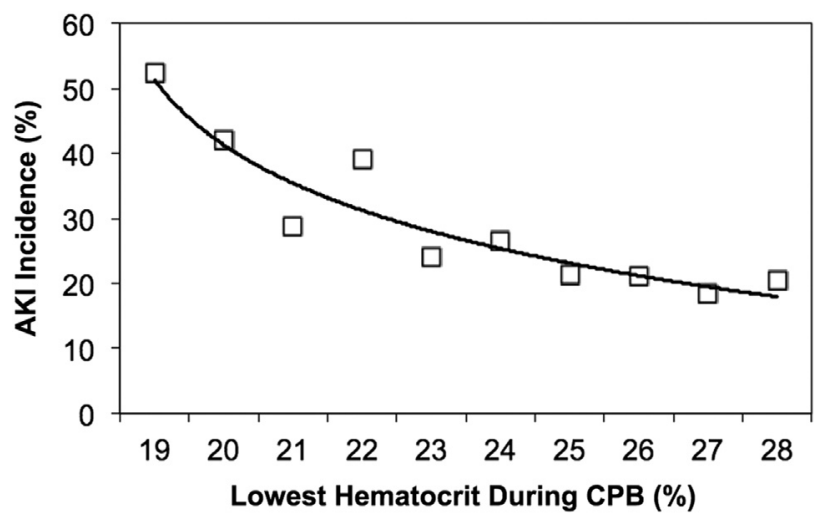

FIGURE 1. Acute kidney injury $(A K I)$ incidence against lowest hematocrit during cardiopulmonary bypass $(C P B)$ for study cohort with logarithmic trend line.

\section{Intraoperative Transfusion}

A total of 501 patients met the transfusion guidelines. However, only 442 patients ( $30.5 \%$ of study population) underwent transfusion. Of these 442 patients, $43.5 \%$ developed AKI. The age of the RBCs used for transfusion was $10.7 \pm 7.8$ days. Transfusion was, however, not independently associated with the development of AKI in our study (RR, 0.961; 95\% CI, 0.782-1.180; $P=.702$; Figure 2).

\section{Other Factors}

The other factors that increased the risk of developing AKI included older patient age ( $\geq 70$ years; RR, 1.350; 95\% CI, 1.085-1.679; $P=.007)$, larger body mass index (RR, 1.053; 95\% CI, 1.030-1.077; $P<.001$ ), history of hypertension (RR, 1.355; 95\% CI, 1.038-1.768; $P=.025$ ), higher preoperative creatinine value $(\mathrm{RR}, 1.003 ; 95 \% \mathrm{CI}$, 1.001-1.006; $P=.016$ ), intra-aortic balloon pump use (RR, 1.480; 95\% CI, 1.184-1.895; $P<.001$ ), and longer CPB time (RR, 1.003; 95\% CI, 1.002-1.004; $P<.001$; Table 2).

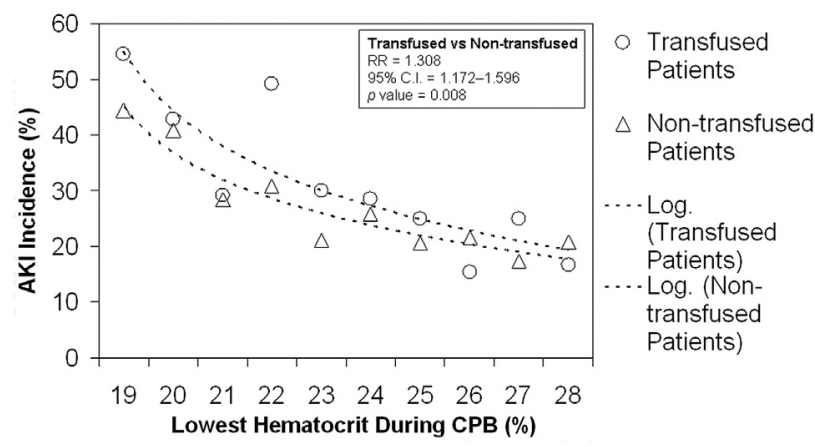

FIGURE 2. Acute kidney injury $(A K I)$ incidence against lowest hematocrit during cardiopulmonary bypass $(C P B)$ for transfused and nontransfused patients in study cohort with logarithmic trend lines. $R R$, Relative risk; $C I$, confidence interval. 
TABLE 2. Multivariate analysis of perioperative risk factors associated with AKI

\begin{tabular}{|c|c|c|c|}
\hline Variable & $\mathbf{R R}$ & $\mathbf{9 5} \% \mathrm{CI}$ & $P$ value \\
\hline \multicolumn{4}{|l|}{ Preoperative } \\
\hline Age $(\geq 70 y)$ & 1.350 & $1.085-1.679$ & .007 \\
\hline Body mass index $\left(\mathrm{kg} / \mathrm{m}^{2}\right)$ & 1.053 & $1.030-1.077$ & $<.001$ \\
\hline History of diabetes mellitus & 1.074 & $0.897-1.285$ & .438 \\
\hline History of hypertension & 1.355 & $1.038-1.768$ & .025 \\
\hline Preoperative anemia & 1.225 & $1.022-1.468$ & .028 \\
\hline Preoperative creatinine $(\mu \mathrm{mol} / \mathrm{L})$ & 1.003 & $1.001-1.006$ & .016 \\
\hline Loop diuretic medication & 1.203 & $0.960-1.506$ & .108 \\
\hline $\mathrm{LVEF}<30 \%$ & 1.162 & $0.886-1.523$ & .278 \\
\hline Urgent or emergent surgery & 1.094 & $0.866-1.383$ & .450 \\
\hline Use of IABP & 1.480 & $1.184-1.851$ & $<.001$ \\
\hline Logistic EuroSCORE & 1.002 & $0.988-1.017$ & .752 \\
\hline \multicolumn{4}{|l|}{ Intraoperative } \\
\hline Lowest hematocrit during CPB (\%) & 0.933 & $0.899-0.968$ & $<.001$ \\
\hline $\mathrm{RBC}$ transfusion & 0.961 & $0.782-1.180$ & .702 \\
\hline CPB time (min) & 1.003 & $1.002-1.004$ & $<.001$ \\
\hline Aortic crossclamp time (min) & 1.000 & $0.9998-1.002$ & .906 \\
\hline Vein grafts harvested (n) & 1.049 & $0.963-1.143$ & .272 \\
\hline
\end{tabular}

\section{In-Patient AKI Morbidity and Mortality}

Of the 391 patients who developed AKI, 19 (4.9\% of the AKI population) developed a new need for dialysis after CABG. Of these 19 patients, 5 subsequently died (mortality $26.3 \%)$.

\section{DISCUSSION}

The incidence of AKI in our study was $27.0 \%$. This was at the higher end of the range $(1 \%-30 \%)$ in published studies from Europe and North America. ${ }^{1,2}$ The lowest hematocrit and preoperative anemia were both independent factors for the development of post-CPB AKI.

\section{Lowest Hematocrit and AKI Incidence}

The lowest hematocrit during CPB is a reliable indicator of the degree of hemodilution. The kidneys are vulnerable to changes in renal blood flow because their metabolism is flow-dependent. ${ }^{9}$ However, hemodilution increases kidney perfusion, but it also causes an increase in the energy requirement of kidneys because of increased tubular transport. This, coupled with the decreased oxygen delivery due to hemodilution and the physiologic hypoxic environment of the renal medulla, can precipitate AKI injury, especially in the oxygen-sensitive renal medulla. ${ }^{2,5}$

In our study, the lowest hematocrit during $\mathrm{CPB}$ was identified as 1 of the potentially modifiable risk factors for our Asian patients undergoing CABG surgery. The lowest hematocrit during $\mathrm{CPB}$ in our patient population of $24.5 \%$ $\pm 3.8 \%$ corroborated with that of previous studies. ${ }^{4,5,9}$
The incidence of AKI in our cohort increased with a decreasing lowest hematocrit during CPB. It was at the lowest hematocrit of $\leq 22 \%$ at which the AKI incidence increased more dramatically (Figure 1). Our findings were in agreement with published studies that have shown the association of lowest hematocrit during $\mathrm{CPB}$ with poorer renal outcomes. ${ }^{4,9}$ Habib and colleagues ${ }^{4}$ showed that the renal failure rates increased as the lowest hematocrit during CPB decreased, in particular when the lowest hematocrit value was $<22 \%$. Karkouti and colleagues ${ }^{9}$ showed that when the lowest hematocrit during CPB was $<21 \%$, the odds of developing acute renal failure with the need for dialysis was 2.34 times greater than when lowest hematocrit during CPB was maintained at $21 \%$ to $25 \%$.

The mean body mass index in our cohort was $25.2 \pm 3.8 \mathrm{~kg} /$ $\mathrm{m}^{2}$. Western studies have reported that the mean body mass index of their patients ranged from 28.2 to 29.2 . $^{16,17}$ Because Asians are smaller in body size, the use of standard CPB circuits designed for Western populations could have resulted in greater hemodilution. However, the lowest hematocrit values during CPB ranged from $14 \%$ to $47 \%$ in our study, similar to that reported by other studies. ${ }^{4,9}$ This was likely because of the liberal use of blood transfusion during CPB to keep an hematocrit of $\geq 21 \%$ in our practice.

\section{Preoperative Anemia and AKI Incidence}

Anemia contributes to AKI by the reduction of renal oxygen delivery and impaired hemostasis. ${ }^{6}$ Coupled with the increased risk of bleeding because of CPB-related hemostatic defects, anemia-induced platelet dysfunction can lead to excessive bleeding during cardiac surgery. This, in turn, will necessitate a greater rate of transfusion, further aggravating the risk of AKI. ${ }^{6}$

In our study, preoperative anemia has been identified as 1 of the potentially modifiable risk factors for our Asian patients undergoing $\mathrm{CABG}$ surgery. Preoperative anemia increased the risk of AKI by $23 \%$, corroborating the findings of published studies. ${ }^{6,7,10}$ Asians with a smaller body size and preoperative anemia will be particularly prone to excessive hemodilution and subsequent blood transfusion-a double insult that could explain the high rate of blood transfusion and risk of AKI in this population.

The patients with preoperative anemia who developed AKI had a mean lowest hematocrit of $21.6 \% \pm 2.6 \%$. This is within the target hematocrit level during $\mathrm{CPB}$ and suggests that the target level of hematocrit might well differ between anemic and nonanemic patients in terms of reducing the AKI risk.

\section{Intraoperative Transfusion and AKI Incidence}

Of our patients, $30.5 \%$ were transfused intraoperatively. The transfusion rate was about 2 times greater than that in other reported series. ${ }^{18,19}$ Transfusion was, however, not independently associated with postoperative AKI 
development in our study (RR, 0.961; 95\% CI, $0.782-1.180 ; P=.702$ ). Transfusion did not play a role in influencing AKI outcomes as significant as that for the lowest hematocrit during CPB (Figure 2). This was surprising, because published data have shown that blood transfusion during CPB in itself can contribute to the risk of AKI by exacerbating the $\mathrm{CPB}$-initiated systemic inflammatory response syndrome with a second inflammatory response, further contributing to ischemia-reperfusion injury in the kidneys. ${ }^{20}$ It can be postulated that blood transfusion in our population partially ameliorated the deleterious effects of the low hematocrit during CPB. This has confirmed that avoiding excessive hemodilution should be the primary objective in the avoidance of postoperative AKI in Asian patients undergoing $\mathrm{CABG}$ surgery with $\mathrm{CPB}$, even if the liberal use of transfusion is required.

The present study had several strengths. It was a prospective study with clearly defined data points. The study population was relatively homogenous, because all patients had easy access to subsidized healthcare. The present study was protocol-driven, with uniform practice among the various anesthetists, perfusionists, and surgeons, ensuring the reliability of the data.

One of the limitations of the present study was that the patients who needed intraoperative blood transfusion to maintain a hematocrit of $\geq 21 \%$ were not differentiated. These patients typically included those with a low body weight or preoperative anemia. The former group of patients will be subjected to acute hemodilution and the latter will already have compensatory mechanisms in place because of the pre-existing anemia. The pathogeneses leading to AKI in these 2 groups of patients are likely to be different. ${ }^{11}$ Future analyses to differentiate these 2 groups of patients will lead to a better understanding of the response of these patients to CPB-induced hemodilution and the risk of AKI. This will allow modification of the management during $\mathrm{CPB}$ using a dual strategy (eg, the use of mini-CPB circuits $^{21}$ for low body weight patients and preoperative treatment of anemic patients using agents such as erythropoietin ${ }^{22}$ ). Through such strategies, 2 potentially modifiable AKI risks could be significantly reduced.

Another limitation of the present study was the lack of data on the patients' hydration status. Dehydration and fluid overload can result in elevation or reduction of a patient's hematocrit, respectively. However, most of our patients were hemodynamically stable and had undergone elective isolated $\mathrm{CABG}$. Thus, the lowest hematocrit during $\mathrm{CPB}$ largely resulted from hemodilution effects or blood loss during surgery and not the patient's hydration status.

\section{CONCLUSIONS}

The lowest hematocrit during CPB and preoperative anemia are 2 potentially modifiable independent risk factors that should be optimized to reduce the incidence of AKI during cardiac surgery in the Asian population. Intraoperative transfusion was not independently associated with postoperative AKI development, and blood transfusion to maintain the hematocrit at $\geq 21 \%$ appeared not to be deleterious. Our results indicate that treatment of preoperative anemia and maintenance of a hematocrit of $\geq 21 \%$ should be used as strategies to reduce the incidence of AKI after $\mathrm{CABG}$ in the Asian population.

\section{References}

1. Conlon PJ, Stafford-Smith M, White WD, Newman MF, King S, Winn MP, et al. Acute renal failure following cardiac surgery. Nephrol Dial Transplant. 1999;14: 1158-62.

2. Rosner MH, Okusa MD. Acute kidney injury associated with cardiac surgery Clin J Am Soc Nephrol. 2006;1:19-32.

3. Haase M, Bellomo R, Story D, Letis A, Klemz K, Matalanis G, et al. Effects of mean arterial pressure, haemoglobin and blood transfusion during cardiopulmonary bypass on post-operative acute kidney injury. Nephrol Dial Transplant. 2012;27:153-60.

4. Habib RH, Zacharias A, Schwann TA, Riordan CJ, Durham SJ, Shah A. Adverse effects of low hematocrit during cardiopulmonary bypass in the adult: should current practice be changed? J Thorac Cardiovasc Surg. 2003;125:1438-50.

5. Swaminathan M, Phillips-Bute BG, Conlon PJ, Smith PK, Newman MF, Stafford-Smith M. The association of lowest hematocrit during cardiopulmonary bypass with acute renal injury after coronary artery bypass surgery. Ann Thorac Surg. 2003;76:784-92.

6. Karkouti K, Wijeysundera DN, Yau TM, Callum JL, Cheng DC, Crowther M, et al. Acute kidney injury after cardiac surgery: focus on modifiable risk factors. Circulation. 2009; 119:495-502.

7. Karkouti K, Wijeysundera DN, Beattie WS. Reducing Bleeding in Cardiac Surgery (RBC) Investigators. Risk associated with pre-operative anemia in cardiac surgery: a multicenter cohort study. Circulation. 2008;117:478-84.

8. Kulier A, Levin J, Moser R, Rumpold-Seitlinger G, Tudor IC, Snyder-Ramos SA, et al. Impact of preoperative anemia on outcome in patients undergoing coronary artery bypass graft surgery. Circulation. 2007;116:471-9.

9. Karkouti K, Beattie WS, Wijeysundera DN, Rao V, Chan C, Dattilo KM, et al. Hemodilution during cardiopulmonary bypass is an independent risk factor for acute renal failure in adult cardiac surgery. J Thorac Cardiovasc Surg. 2005; 129:391-400.

10. Khanna MP, Hebert PC, Fergusson DA. Review of the clinical practice literature on patient characteristics associated with perioperative allogeneic red blood cell transfusion. Transfus Med Rev. 2003;17:110-9.

11. Karkouti K, Wijeysundera DN, Yau TM, McCluskey SA, Chan CT, Wong PY, et al. Influence of erythrocyte transfusion on the risk of acute kidney injury after cardiac surgery differs in anemic and nonanemic patients. Anesthesiology. 2011; 115:523-30.

12. World Health Organization. Public health appropriate body-mass index for Asian populations and its implications for policy and intervention strategies. Lancet. 2004;363:157-63.

13. Nadler SB, Hidalgo JU, Bloch T. Prediction of blood volume in normal human adults. Surgery. 1962;51:224-32.

14. Couchoud C, Pozet N, Labeeuw M, Pouteil-Noble C. Screening early renal failure: cut-off values for serum creatinine as an indicator of renal impairment. Kidney Int. 1999;55:1878-84.

15. Chew ST, Mar WM, Ti LK. Association of ethnicity and acute kidney injury after cardiac surgery in a South East Asian population. Br J Anaesth. 2013;110: 397-401.

16. Brown JR, Kramer RS, Coca SG, Parikh CR. Duration of acute kidney injury impacts long-term survival after cardiac surgery. Ann Thorac Surg. 2010;90:1142-8.

17. Perez-Valdivieso JR, Monedero P, Vives M, Garcia-Fernandez N, Bes-Rastrollo M. GEDRCC (Grupo Español de Disfunción Renal en Cirugía Cardiaca). Cardiac-surgery associated acute kidney injury requiring renal replacement therapy: a Spanish retrospective case-cohort study. BMC Nephrol. 2009; 10:27.

18. Che M, Li Y, Liang X, Xie B, Xue S, Qian J, et al. Prevalence of acute kidney injury following cardiac surgery and related risk factors in Chinese patients. Nephron Clin Pract. 2011;117:c305-11. 
19. Vellinga S, Verbrugghe W, De Paep R, Verpooten G, Janssen van Doorn K. Identification of modifiable risk factors for acute kidney injury after cardiac surgery. Neth J Med. 2012;70:450-4.

20. Fransen E, Maessen J, Detener M, Senden N, Buurman W. Impact of blood transfusions on inflammatory mediator release in patients undergoing cardiac surgery. Chest. 1999;116:1233-9.
21. Benedetto U, Luciani R, Goracci M, Capuano F, Refice S, Angeloni E, et al Miniaturized cardiopulmonary bypass and acute kidney injury in coronary artery bypass graft surgery. Ann Thorac Surg. 2009;88:529-35.

22. Song YR, Lee T, You SJ, Chin HJ, Chae DW, Lim C, et al. Prevention of acute kidney injury by erythropoietin in patients undergoing coronary artery bypass grafting: a pilot study. Am J Nephrol. 2009;30:253-60. 